\title{
Dominant Processes Concept, Model Simplification and Classification Framework in Catchment Hydrology
}

\author{
B. Sivakumar ${ }^{1,2}$ \\ ${ }^{1}$ Department of Land, Air and Water Resources, University of California, Davis, CA 95616, USA \\ ${ }^{2}$ Griffith School of Engineering, Griffith University, Nathan, QLD 4111, AUSTRALIA \\ E-mails: sbellie@ucdavis.edu; s.bellie@griffith.edu.au
}

Keywords: Catchment modeling, Simplification, Dominant processes, Classification framework, Terminology

\section{EXTENDED ABSTRACT}

Technological and methodological advances have facilitated tremendous growth in hydrology during the last century. Invention of powerful computers, remote sensors, geographic information systems, and worldwide networking have facilitated extensive data collection (both in time and in space), better data sharing, formulation of sophisticated methods (including those for studying the inherent hydrologic nonlinearities and scalings), and development of highly complex models. There is no question that we today have a far greater ability to mimic real hydrologic systems and possess a much better understanding and predictive ability of hydrologic phenomena than we did not long ago.

While this situation is indeed encouraging, there are concerns that the same technological and methodological advances also indirectly contribute to additional problems in hydrologic research. An insight into hydrologic literature clearly reveals our tendency to develop more complex models (having too many parameters, thus requiring too much data) than perhaps needed and our increasing emphasis on individual mathematical techniques for specific situations rather than their collective utility for common hydrologic issues. There has been increasing realization in recent times that simplification in modeling and development of a common framework for hydrology is necessary to alleviate these problems, and a number of studies have indeed advocated and argued for such a research practice (e.g. Dooge 1986; Young et al. 1996; Grayson and Blöschl 2000; Sivakumar 2000, 2004; Beven 2002; Woods 2002; Sivapalan 2005; Dawdy 2007). Unfortunately, however, these studies are of diverse forms, and efforts to bring these mostly disparate studies together to find common grounds are almost non-existent.

In view of this, the present study is intended to bring the above studies together towards a more coherent approach to research in catchment hydrology. This is done by: (1) reviewing the current status of hydrologic research and highlighting the need for model simplification and generalization; and (2) proposing some potential directions to achieve such. Through a discussion of general/specific characteristics of hydrologic 'systems' (actual and perceived complexity as well as actual and potential simplicity) and difficulties in data measurements, the need for moving beyond the notion of 'modeling everything' to the notion of 'capturing the essential features' is explained; the concept of dominant processes in model simplification and the utility of integration of different concepts and methods for modeling improvement are discussed. Formulation of a catchment classification system is advocated as a possible means for a common framework in hydrology, and the role of dominant processes in this formulation is presented; the problems due to adoption of different modeling terminologies are highlighted and potential ways to overcome such are also discussed.

While the main focus of the present study is on issues related to simplification and generalization, other issues that play critical/relevant roles in hydrologic research are also addressed. The results of the study reflect an important paradigm change that is needed to attain further progress in hydrology, as follows. In the current modeling practice, complex models and individual mathematical techniques are the norm, while simplification and generalization are only the exception. In view of the difficulties that lie with this practice, the present study advocates adoption of a new practice where simplification and generalization are the norm, while complex models and individual techniques are only the exception. The review of the current research status and proposal of new directions presented herein clearly demonstrate the need and scope for this change. It is hoped that the study would lead to a better research and teaching philosophy in hydrology. 


\section{INTRODUCTION}

Hydrology has witnessed an enormous growth in the twentieth century, thanks to technological and methodological advances. Invention of powerful computers, remote sensors, geographic information systems, and networking have facilitated extensive data collection, better data sharing, formulation of sophisticated methods, and development of complex models. There is no doubt that we today have a far greater ability to mimic real hydrologic systems than we did not long ago.

While technological and methodological advances are definitely needed for a more complete pathway to deal with hydrologic systems, it is important to recognize that true progress must be evaluated only through a balanced assessment of their positive (benefit) and negative (cost) aspects. However, encouraged by their positive aspects, we have a tendency to simply continue using them and potentially overlook their limitations. As a result, it is fair to say, in the context of true progress (rather than pure development, i.e. developing slightly different and more complex models), that there is an imbalance in our dealing with hydrologic systems. An examination of literature reveals that there exist serious problems in the ways we approach the modeling task. There are concerns that: (1) these technological advances naturally lead to more complex models (having too many parameters, requiring too much data) than that may actually be needed; and (2) these models are often developed for specific situations, thus making generalization difficult. There are also criticisms that: (1) we may not be collecting all the relevant data, though collecting more data; and (2) despite their complexities, these models often do not perform sufficiently well, even the same situations they are developed for (e.g. Beven 2002).

The above remarks emphasize the urgent need to study two critical issues for true progress in hydrology: (1) simplification of modeling and collection of more reliable data; and (2) development of a common modeling framework. These issues have indeed been part of our current research: the ideas of model simplification and collection of more reliable data are central in many studies (e.g. Young et al. 1996; Grayson and Blöschl 2000; Sivakumar 2000; Beven 2002), and the need for a common framework has been recognized by many researchers (e.g. Dooge 1986; Woods 2002; Sivakumar 2004; Sivapalan 2005; Dawdy 2007). The problem, however, is that efforts to bring these mostly disparate studies together to find common grounds are non-existent. Two aspects, among others, are likely causing this situation: (1) our tendency to develop increasingly complex models, with the belief that the more complex the models, the better the outcomes; and (2) our increasing emphasis on individual mathematical techniques for analyzing specific data rather than their collective utility for solving common hydrologic problems. These aspects are further explained, through examples, as follows.

Proposing hypothesis testing in hydrology, Beven (2001) highlights the role of highly complex models as a possible hindrance to progress, with specific reference to the problems faced by graduate students. In one of the four hypotheses, he proposes: "Graduate students can always think of useful new hypotheses with which to test models (rather than simply calibrating existing models and learning little)." However, he subsequently comments: "The literature does not give the impression that this hypothesis is being tested with any enthusiasm [at the current time]. It seems to be the case that graduates are being taught more about how to use models than about how to critically evaluate models. This is hardly surprising given the complexity and learning curves necessary for some of the models [that are currently available], and being able to use models is a precursor to being able to criticize them."

The study by Sivakumar (2005) highlights the negative effect of our increasing emphasis on individual techniques that serve mostly 'the specialists,' rather than a more generalized approach that would serve everyone. Discussing the role of 'thresholds' in hydrologic systems and the various implicit forms in which they are already being adopted, Sivakumar (2005) comments: "Looking at the direction in which hydrologic research is moving, with our emphasis on specific data analysis techniques than common hydrologic problems [an opinion may not be shared by everyone], we could end up with enormous difficulties in understanding hydrologic literature [dealing with thresholds]. This is immediately evident [from the above examples], since [I suspect] not many hydrologists who are familiar with the concept of self-organized criticality are also familiar with the concept of artificial neural networks and/or nonlinear deterministic and chaos theories. It is obvious that the [above] situation would only become worse when hydrologic concepts, research activities and model success/failure need to be explained and disseminated to new and emerging hydrologists (e.g. undergraduate and graduate students), water managers and policy makers.”

In view of these issues, an attempt is made in the present study to highlight the need for model simplification and generalization and also to 
propose some potential directions to achieve such. While the main focus is on issues related to simplification and generalization in catchment hydrology, other issues that play critical roles in hydrologic research are also addressed.

\section{HYDROLOGIC SYSTEMS AND CURRENT MODELING TREND}

Hydrologic processes arise as a result of interactions between climate inputs and landscape characteristics that occur over a wide range of space and time scales. Due to the heterogeneities in landscape properties and climatic inputs, such processes may be highly variable and complex at all scales (although simplicity is possible). Therefore, it would be useful, or even necessary, to measure the relevant data at all of these scales for a complete understanding and/or prediction of these processes. However, it is not practical to routinely observe the processes at all these scales. Therefore, observations are made only at the catchment scale or at a few chosen scales; similar is the situation when considering time scale. This necessitates understanding and/or 'transforming' the process of interest at the scale of interest from the corresponding process at another scale. Extensive details of scale issues in hydrology are already available in the literature (e.g. Gupta and Waymire 1990; Blöschl and Sivapalan 1995). Furthermore, our purpose need not always be 'understanding' of the processes (though this is desired) but rather at times 'prediction' for tackling specific potential immediate problems (e.g. floods). Dawdy (2007) presents an excellent account of this 'prediction versus understanding' issue, pointing out that: “... understanding does not necessarily lead to better prediction. Prediction is an engineering problem. Understanding, in general, is a scientific problem.” What we can hope for the future, however, is to develop theories or other explanations, where understanding may, in fact, aid in prediction..

Considering the above, hydrologic 'systems' may be viewed from three different angles: process, scale, and purpose of interest. Depending upon the angle at which they are viewed, hydrologic systems may be either simple or complex; consequently, the appropriate models to represent a given system may also be either simple or complex. The obvious question then is: how simple or how complex the models should be? Granted that there can be numerous hydrologic systems even within a given catchment, not all of these may actually define (or be necessary for) the catchment. However, in our ambition to know everything (i.e. to model everything), we try to gather more and more information in the form of data and incorporate such information in our conceptual thinking, i.e. developing more and more complex models. This process requires additional technological and human resources. Moreover, these efforts themselves give rise to additional difficulties; for example, collection of additional data may require sophisticated measurement devices, but that is not the end of the story, since to analyze such data we may also require better mathematical and computational tools. Once better tools are found, then they in turn become the 'driving force' for collection of even more data and development of even more complex models. This kind of 'vicious circle' but often with increasing 'complexification' is the existing trend.

While there is no philosophical flaw in this line of thinking, there are many realistic flaws:

1. Even if the intended purpose of our modeling exercise is defined clearly, there is often lack of knowledge about the type of measurements to be made, since the physical mechanisms governing hydrologic systems may be unknown. In fact, one of the purposes of measuring data is to learn about such mechanisms from the data themselves in an 'inverse' manner.

2. In spite of our technological advances, there are still many constraints in regards to data measurements, some of which are associated with such technologies themselves. For example, while remote sensing has facilitated precipitation estimation at different scales, such still requires raingage measurements (i.e. 'ground truth') for such estimation. However, since raingage measurements themselves are contaminated with errors, precipitation estimation from remote sensors will also contain errors. Even if these errors happen to be small, they may still result in large effects in the final outcomes, a property called 'sensitivity to initial conditions’ (e.g. Lorenz 1963); and

3. Even if we have all the right technologies for data measurements, we still may not be able to actually obtain the required data for reasons associated with political and societal pressures. The difficulties in obtaining accurate data for rivers shared by two or more countries/states is an excellent example for this situation.

In view of these, we must remember two things: (1) we may not always be able to get the data we need; and (2) even if we succeed in getting, what we get is not necessarily what it actually is. Consequently, our modeling exercises and capabilities suffer. The effects of inadequate/inaccurate data on our modeling are extensively documented in the literature in diverse forms, including overparameterization, equifinality, uncertainty, uniqueness, and sensitivity to initial conditions. 


\section{MODEL SIMPLIFICATION AND DOMINANT PROCESSES CONCEPT}

An essential first step in addressing the limitations of our current approach to modeling is to acknowledge their existence, i.e. admitting that our 'modeling everything' notion is somewhat flawed, and that complexification may not always be the way to go. While this is not an easy thing to do, the recent surge in such acknowledgments is encouraging. For example, the studies by Young et al. (1996), Grayson and Blöschl (2000) and Woods (2002) advocate, in one way or another, moving beyond the notion of 'modeling everything' and adopting the notion of 'capturing the essential features.” Their argument, in essence, is: “... we should be developing methods to identify dominant processes that control hydrologic response and then developing models to focus on these dominant processes.”

Regardless of the name this idea of simplification assumes in the literature, whether 'dominant process concept' (Grayson and Blöschl, 2000) or another [see Sivakumar (2004)], it emphasizes the need and our willingness to compromise, in the face of reality. The dominant processes concept has a particular advantage in that it helps to be effective and efficient in real time, since it requires only less data collection, but more important and reliable. It would also provide a framework for the development and application of techniques especially designed to deal with those [dominant hydrologic] controls and help to avoid some of the overparameterization problems that occur when processes that are not important are represented.

In addition to the above data-constraint perspective, the idea of model simplification has two other important facets: (1) our knowledge through observations that often only a few processes dominate hydrologic response in a given catchment; and (2) our experience through modeling, parameter estimation, and prediction that simple models with only a few dominant parameters could capture the essential features of a given catchment's response to hydrologic events. The case studies in Grayson and Blöschl (2000), representing a range of environments, dominant processes, catchment sizes, data types and modeling techniques, provide support to these by revealing that a single process dominates the response in the studied catchments. Hydrologic literature is replete with further support to this.

While the realization that consideration of only one or a few dominant processes may be sufficient for modeling is encouraging, determination of this 'number' and the identification of the processes are not straightforward. A logical way to deal with this is by evaluating the sensitivity of the system to each of the individual processes that are believed to have influence. This procedure starts with the 'most possible complex situation' (i.e. combination of all processes) and moves towards the 'simplest reliable solution' (i.e. combination of dominant processes), through a trial-and-error elimination method. This procedure has been the cornerstone of conventional sensitivity analysis and parameter estimation studies. Although generally reliable, this procedure is enormously expensive from the perspectives of data, time and computer requirements. In cases where a much larger number of influencing processes are involved, the conventional procedure may be of little use and even doomed to failure (e.g. Sivakumar 2004).

These observations clearly reflect the need for a better procedure for identification of the dominant processes. One possible way to achieve this is through a procedure that starts with the 'simplest reliable situation' and moves towards the 'most complex potentially required solution.' This does not mean that one must always start with just one process and include additional ones as needed. Rather, it means that one must find a suitable method to first reliably determine the number of dominant processes from only the available (often limited) data, so that this number can serve as a reliable starting point.

Numerous methods already exist, especially in mathematics/physics literature, for determining the number of dominant processes from only limited available data; many of these methods require only a single-variable series that is representative of the system. These methods are mostly rooted in the concept of 'dimension' and involve representation of the multi-dimensional system using a singlevariable series through a 'pseudo' state-space reconstruction, popularly known as 'phase-space reconstruction' (e.g. Packard et al. 1980). The idea behind this reconstruction is that a nonlinear system is characterized by self-interaction, and that a series of a single variable can carry the information of the dynamics of the multi-variable system. There is ample proof in the literature for the appropriateness and usefulness of this data reconstruction concept for systems that are highly nonlinear and complex as that of fluid turbulence and weather weather. Since hydrologic systems have long been known to be inherently nonlinear, this kind of reconstruction could be a useful tool for determining the number of dominant processes.

The existing methods based on the phase-space reconstruction concept for dominant processes 
may be grouped under two categories: 'dimension' methods and 'prediction' methods. While the former category can estimate the dimensionality of the system in a 'direct' way, in the latter prediction is first made and dimensionality is subsequently obtained in an 'inverse' manner. The correlation dimension method is an example for the former, and the nonlinear local approximation method is an example for the latter. These methods have widely been employed for the determination of the number of dominant processes in different hydrologic systems, especially in the context of nonlinear deterministic dynamics. While caution is needed in employing these methods to real data, the outcomes of such studies are generally reliable (e.g. Sivakumar 2000). However, there is a difficulty in using these methods to deal with dominant processes; they only indicate the number of dominant processes but do not identify the actual processes. Therefore, we must find a way to advance these methods to achieve our larger goal of identifying the dominant processes. This remains a great challenge at the current time since none of the existing methods seems capable of handling this task by itself. Consequently, integration of different concepts and methods seems to be the only way to address this problem.

\section{MODELING IMPROVEMENT - INTEGRATING CONCEPTS}

It is common in our current research to focus on specific methods to address specific hydrologic issues. While this is understandable, this also comes with a price. Since each of these methods has limitations, it would be unwise of us to think that we can solve a given problem using one particular method. Further, since each of these methods often possesses different advantages and limitations, it would be sensible of us to attempt to maximize their advantages and minimize limitations. One possible means to do this is through integrating two or more methods.

One such integration approach to deal with the identification of dominant processes is presented in Sivakumar (2004). This approach attempts integration of three different concepts (nonlinear determinism, expert advice, and parameter optimization) and involves three steps: (1) determination of the number of dominant processes governing the system, using the correlation dimension method; (2) identification of dominant processes, through expert knowledge; and (3) sensitivity analysis to arrange the dominant processes as per their extent of dominance. These steps have advantages of requiring data of only a single variable representing the system, honoring field reality, and significantly reducing data collection, time and computer costs. Since the correlation dimension method provides a reliable estimate of the number of dominant processes, this procedure can be very efficient, as it starts with the 'simplest reliable situation' and moves towards the 'most complex potentially required solution,' if at all inclusion of additional information is needed.

It must be noted that the proposal in Sivakumar (2004) may be just one example of possible integrations to identify the dominant processes, and there may be many other possibilities involving either the very same concepts or others. In view of these, regardless of the problem one looks at, one thing seems to be clear: it is important for us to not only be aware of the different existing concepts/methods but also have a 'general' understanding of them so that we will be in a position to choose the appropriate ones for integration in the first place. Unfortunately, this task is extremely challenging, considering our tendency to focus on specific techniques rather than finding common grounds. In view of these, there is indeed a need to work towards some kind of generalization and a common framework in hydrologic research.

\section{HYDROLOGIC CLASSIFICATION - ROLE OF DOMINANT PROCESSES}

Since hydrologic systems may exhibit widely varying levels of complexity, there is a need for a framework that could help in determining the extent of complexity, so that an appropriate identification as to the (type of) model and data requirements may be made. This is the motivation behind the calls for a hydrologic classification system (e.g. Woods 2002). For instance, a chaotic model may be more appropriate for a lowdimensional system, whereas a stochastic model may be more appropriate for a high-dimensional system. As noted in McDonnell and Woods (2004), classification of phenomena is (and must be) a standard first step in the process of scientific analysis and synthesis.

Obviously, such a classification framework must be able to provide guidelines for streamlining hydrologic system complexity into classes/subclasses, based on the general/specific information available. For its usefulness to be realized both at the global and at the local/regional levels, the framework must be developed so as to accommodate important general (e.g. process) as well as specific (e.g. catchment) characteristics of the hydrologic systems. The framework must also be simple enough and commonly agreeable, so that it could provide a 'universal' language for communications and discussions in hydrology. 
According to some studies (e.g. Woods 2002), identification of dominant processes may help in the formation of a classification system in hydrology. This is the idea behind the classification system introduced in Sivakumar (2004), in which the 'extent of complexity' or 'dimensionality' of hydrologic systems is treated as a representation of the (number of) dominant processes. Following up on this, Sivakumar et al. (2007) explore the utility of phase-space reconstruction concept, in which the 'region of attraction of trajectories' in the phase-space is used to identify the data as exhibiting 'simple' or 'intermediate' or 'complex' behavior and, correspondingly, classify the system as potentially low-, medium- or high-dimensional. The utility of this concept is first demonstrated on two artificial time series possessing significantly different characteristics and levels of complexity, and then tested on a host of river-related data representing different geographic regions, climatic conditions, basin sizes, processes and scales. Yielding 'attractors' that range from 'very clear' ones to 'very blurred' ones depending on the studied data (i.e. 'system'), the results indicate the usefulness of this simple reconstruction concept for studying hydrologic system complexity and classification.

While these outcomes reveal reveal the utility of the phase-space reconstruction and other databased concepts, this is only a partial job done. What remains is establishing connections between the measured data (usually at the catchment scale) and actual physical mechanisms (at various scales). While there have indeed been attempts to establish these connections for long (e.g. Klemes 1978), a coherent approach to deal with this problem remains elusive. In this regard, current efforts that attempt to reconcile the upward (process-based) and downward (data-based) approaches (e.g. Sivapalan 2005) are certainly encouraging. Also, scaling theories could certainly lead to a better understanding of hydrologic systems and processes and potentially to better predictions, as noted by Dawdy (2007). However, research in these directions are still at the state of infancy.

\section{EXISTING CONFUSION AND REQUIRED GENERALIZATION - THE CASE OF TERMINOLOGY}

Adopting different terminologies that represent similar ideas and procedures has become a common problem in hydrology. This, I believe, has resulted essentially due to our emphasis on specific concepts and methods rather than on common hydrologic issues. The study by Sivakumar (2005) explains this situation with an example of the role of 'thresholds' in hydrologic systems and the various implicit forms it takes in hydrologic literature depending upon the method/area of study, such as 'critical states' in studies on self-organization and criticality, 'characteristic patterns' in studies on selforganizing maps and artificial neural networks, and 'regimes' in studies on nonlinear determinism and chaos and in others, in addition to the explicit form of 'thresholds' it takes. The difficulties associated with these different terminologies are very clear, since not many of us are familiar with all of these concepts. The situation is not very different when it comes to the definitions and modeling procedures adopted under different areas/methods of hydrologic research, or even within the same. For example, the terms validation and verification are used with different, and sometimes interchangeable, meanings in different studies, whether prediction studies using artificial neural networks or parameter estimation using optimization procedures.

In view of the above, there is indeed an urgent need to address this issue and follow some kind of generalization in our modeling practice. An obvious way to achieve this is through adoption of common terminologies, definitions, and modeling procedures, that are acceptable to everyone. Some efforts have already been made in this direction. For example, Refsgaard and Henriksen (2004) present an extensive account of the inconsistencies among terminologies used in hydrologic modeling, especially in the optimization procedures. They subsequently propose a framework for quality assurance guidelines, including a consistent terminology and a foundation for a methodology bridging the gap between scientific philosophy and pragmatic modeling. Similarly, the term 'thresholds' advocated by Sivakumar (2005) could be appropriate for everyone to follow, for its generality and simplicity, which are reflected by the analogy between catchments and humans. While these studies (Refsgaard and Henriksen 2004; Sivakumar 2005) are certainly in the right direction towards bringing some kind of generalization, they unfortunately form only an extremely small fraction of studies, with the vast majority still contributing strong to additional terminologies and further confusion. I hope, however, that these studies are precursors to the many that will be coming in the future to push forward the idea of generalization in hydrology.

\section{CONCLUSIONS}

Technological and methodological advances have undoubtedly played a key role in the growth of hydrology. However, they have also led to some additional problems, such as development of more 
complex models than perhaps needed and emphasis on individual mathematical techniques rather than a common framework. While many studies have recognized these problems, they are in diverse forms, and there has been almost no coherent effort to bring them together. The present study was intended to address this issue.

In regards to model simplification, hydrologic realism and our research experience served as inputs to highlight the need and scope for moving beyond the notion of 'modeling everything' and adopting the notion of 'capturing the essential features.' The importance of identifying the dominant processes that govern hydrologic responses in a given system and developing models to focus only on these dominant processes was emphasized. The need for an integrated modeling approach, that goes beyond the use of a single method and can take into account the advantages of different concepts, for identifying dominant processes and for improvements in modeling was also discussed. On the issue of generalization, the difficulties that arise because of our emphasis on specific mathematical techniques rather than hydrologic issues and thus the need for a common modeling framework, were highlighted. As potential directions towards achieving this, formulation of a classification framework, the role of dominant processes in such formulation, and proposal of common terminologies were discussed.

The present study reflects an important paradigm change needed for true progress in hydrology. Looking at the current modeling practice, it is fair to say that complex models and individual mathematical techniques are the norm, while simplification and generalization are only the exception. It would be more appropriate to adopt a new practice where simplification and generalization are the norm, while complex models and individual techniques are only the exception. Through a review of the current research status and proposal of new directions, the study has clearly demonstrated the need and scope for this change.

\section{REFERENCES}

Beven, K.J. (2001), On hypothesis testing in hydrology, Hydrological Processes, 15, 1655-1657.

Beven, K.J. (2002), Uncertainty and the detection of structural change in models of environmental systems, In Beck, M.B. (ed.), Environmental Foresight and Models: A Manifesto, Elsevier Science, pp. 227-250.

Blöschl, G. and M. Sivapalan (1995), Scale issues in hydrological modeling - a review, Hydrological Processes, 9, 251-290.
Dawdy, D.R. (2007), Prediction versus understanding, ASCE Journal of Hydrologic Engineering, 12, 1-3.

Dooge, J.C.I. (1986), Looking for hydrologic laws, Water Resources Research, 22(9), 46S-58S.

Grayson, R.B. and G. Blöschl (2000), Spatial Patterns in Catchment Hydrology: Observations and Modeling, Cambridge University Press.

Gupta, V.K. and E. Waymire (1990), Multiscaling properties of spatial rainfall and river flow distributions, Journal of Geophysical Research, 95(D3), 1999-2009.

Klemes, V. (1978), Physically based stochastic hydrologic analysis, Advances in Hydrosciences, 11, 285-352.

Lorenz, E.N. (1963), Deterministic nonperiodic flow, Journal of the Atmospheric Sciences, 20, 130-141.

McDonnell, J.J. and R. Woods (2004), On the need for catchment classification, Journal of Hydrology, 299, 2-3.

Packard, N.H., J.P. Crutchfield, J.D. Farmer and R.S. Shaw (1980), Geometry from a time series, Physical Review Letters, 45(9), 712716.

Refsgaard, J.C. and H.J. Henriksen (2004), Modeling guidelines - terminology and guiding principles, Advances in Water Resources, 27, 71-82.

Sivakumar, B. (2000), Chaos theory in hydrology: important issues and interpretations, Journal of Hydrology, 227(1-4), 1-20.

Sivakumar, B. (2004), Dominant processes concept in hydrology: moving forward, Hydrological Processes, 18(12), 2349-2353.

Sivakumar, B. (2005), Hydrologic modeling and forecasting: role of thresholds, Environmental Modeling \& Software, 20(5), 515-519.

Sivakumar B, A.W. Jayawardena and W.K. Li (2007), Hydrologic complexity and classification: a simple data reconstruction approach, Hydrological Processes, 21, DOI: 10.1002/hyp6362.

Sivapalan, M. (2005), Pattern, process and function: elements of a unified theory of hydrology at the catchment scale, In: Anderson, M.G. (ed.) Encyclopedia of Hydrological Sciences, John Wiley \& Sons, pp. 193-219.

Woods, R. (2002), Seeing catchments with new eyes, Hydrological Processes, 16, 11111113.

Young, P.C., S.D. Parkinson and M. Lees (1996), Simplicity out of complexity in environmental systems: Occam's Razor revisited, Journal of Applied Statistics, 23, 165-210. 